\title{
Hepatitis $B$ reactivation in psoriasis patients treated with anti-TNF agents: prevention and management
}

\section{Maria Vittoria Cannizzaro \\ Chiara Franceschini \\ Maria Esposito \\ Luca Bianchi \\ Alessandro Giunta}

Department of Dermatology, University of Rome Tor Vergata, Rome, Italy
Correspondence: Alessandro Giunta Department of Dermatology and Venereology, University of Rome Tor Vergata, Viale Oxford, 8I, Rome 00133 , Italy

Tel +3906 20902743

Email alessandro.giunta@uniroma2.it
This article was published in the following Dove Press journal:

Psoriasis: Targets and Therapy

I5 April 2017

Number of times this article has been viewed

\begin{abstract}
The risk of hepatitis $\mathrm{B}$ virus (HBV) reactivation $(\mathrm{HBVr})$ in chronic $\mathrm{HBV}$ carriers, in occult HBV patients or in acute HBV patients affected by psoriasis and treated with antitumor necrosis factor (TNF)- $\alpha$ agents is a clinical practice issue to face with, particularly if the treatment has a long-term maintenance finality. The aims of this review are to examine the current knowledge on HBVr incidence in chronic HBV carriers and potential occult carriers undergoing therapy with biologics for the treatment of psoriasis and psoriatic arthritis; analyze the prophylactic measure to prevent HBV reactivation and define how to manage $\mathrm{HBVr}$ in patients treated with biologics. We searched through PubMed, Google Scholar and Scopus databases and evaluated all published manuscripts concerning HBVr in psoriatic patients, both plaquetype and psoriatic arthritis, in treatment with any indicated anti-TNF- $\alpha$. Although anti-TNFs are considered moderate immunosuppressive drugs, the incidence of HBVr in psoriatic patients is lower compared to patients affected by other immune-mediated diseases treated with TNF inhibitors. HBV prophylaxis should be probably reserved to anti-HBs+/anti-HBc+ patients with a viral load $<2000 \mathrm{IU} / \mathrm{mL}$ and alterations in serum liver enzymes, in order to prevent HBVr.
\end{abstract}

Keywords: HBV reactivation, anti-TNF- $\alpha$, psoriasis, immune-mediated diseases, HBV reactivation prevention, biologics

\section{Introduction}

Hepatitis B virus (HBV) is a hepadnavirus responsible for most of the chronic liver diseases worldwide and can cause both acute and chronic disease. The virus is transmitted through either parenteral, sexual or vertical route. ${ }^{1}$ It has been estimated that 240 million people worldwide are chronically $\mathrm{HBV}$ infected and $>686,000$ people die every year due to complications of hepatitis $\mathrm{B}$, including cirrhosis and hepatocellular carcinoma. ${ }^{2} \mathrm{HBV}$ is highly endemic in China, South East Asia, Indonesia and subSaharan Africa; in these regions, $>8 \%$ of the population is affected by chronic HBV (CHB) infection. South America, South West Asia, Eastern and Southern Europe are identified as intermediate, with a chronic HBV infection rate between $2 \%$ and $7 \%$ of the population. Developed countries, including North America and Western Europe, are grouped as low-endemic regions and HBV prevalence rates range from $0.5 \%$ to $2 \%{ }^{3}$

In the last decades, a number of agents specifically targeting and thus inhibiting the biologic activity of tumor necrosis factor- $\alpha$ (TNF- $\alpha$ ) have been widely used in clinical practice in the treatment of immune-mediated inflammatory diseases, including rheumatoid arthritis, inflammatory bowel diseases and psoriasis, both plaque-type (Pso) and psoriatic arthritis (PsA). ${ }^{4}$ Even though TNF- $\alpha$ is a cytokine playing a pivotal role in the pathogenesis of inflammatory diseases, its role in the immune-mediated 
response to infections, especially against intracellular pathogens, is central. ${ }^{5}$ Indeed, one of the issues that clinicians have to manage in clinical practice is the possibility that the blocking of TNF- $\alpha$ could induce reactivation of chronic or latent infections, particularly HBV hepatitis.

Therefore, it is essential to serologically monitor all psoriatic patients undergoing immunosuppressive or immunomodulant treatments for possible de novo HBV exposition and to strictly follow-up chronic HBV psoriatic patients to reduce $\mathrm{HBVr}$ rates and promptly manage possible $\mathrm{HBV}$ reactivation $(\mathrm{HBVr})$.

Serologic markers for HBV infection include 1) the surface antigen (HBsAg), which represents the hallmark of $\mathrm{HBV}$ infection, 2) the core virus antigen (HBcAg), 3) the envelope antigen ( $\mathrm{HBeAg}$ ) and 4 ) the antibodies against the virus, namely, anti-HBsAg (anti-HBs), anti-HBeAg (anti$\mathrm{HBe}$ ), and anti-HBcAg (anti-HBc) both IgM and IgG.

The differences in positivity or negativity for these markers allows to 1) identify patients with $\mathrm{HBV}$ infection, 2) elucidate the natural course of $\mathrm{CHB}, 3$ ) assess the clinical phases of infection and 4) monitor antiviral therapy. ${ }^{1}$

HBV infection can be differentiated to acute, chronic, and occult. In particular, presence of $\mathrm{HBeAg}$ or anti-HBe in HBsAg-positive patients with viral load (HBV-DNA) $>2000 \mathrm{IU} / \mathrm{mL}$ identifies an active carrier, a condition frequently associated with a hepatic sufferance. ${ }^{6-9}$ The persistence of detectable HBsAg rates for $>6$ months defines a CHB infection. Indeed, the positivity for HBsAg, but not $\mathrm{HBe} A g$, in patients with detectable anti-HBe, serum alanine aminotransferase (ALT) levels within normal ranges and HBV-DNA undetectable or $<2000 \mathrm{IU} / \mathrm{mL}$, for at least 1 year of follow-up, delineate an inactive carrier, a status generally not associated with a significant liver disease. Subjects expressing anti-HBc and anti-HBs antibodies are defined as potential occult carriers: in these patients, we should take into consideration a possible low viral rate replication in the liver, although HBV-DNA could be undetectable in serum (Table 1). ${ }^{6}$ Covalently closed circular HBV-DNA persists in the nucleus of infected hepatocytes and HBV genome can integrate into host genome. These observations could explain the HBV-related oncogenesis and the odd risk to the development of hepatocellular carcinoma..$^{7-9}$

\section{Methods}

We searched through PubMed, Google Scholar and Scopus databases and evaluated all published manuscripts concerning HBVr in psoriatic patients, both plaque-type and PsA, in treatment with any indicated anti-TNF- $\alpha$. In particular, we identified ten reports describing HBVr during adalimumab,
Table I Definition of HBV infection status based on serologic markers and necroinflammatory activity

\begin{tabular}{llll}
\hline Markers & $\begin{array}{l}\text { Active } \\
\text { carrier }\end{array}$ & $\begin{array}{l}\text { Inactive carrier } \\
\text { (chronic infection) }\end{array}$ & $\begin{array}{l}\text { Potential } \\
\text { occult carrier }\end{array}$ \\
\hline $\mathrm{HBsAg}$ & $\checkmark$ & $\checkmark$ & $\times$ \\
Anti-HBs & $x$ & $x$ & $\checkmark$ or $x$ \\
$\mathrm{HBeAg}$ & $\checkmark$ or $x$ & $x$ & $x$ \\
Anti-HBe & $\checkmark$ or $x$ & $\checkmark$ & $\times$ \\
Anti-HBc & $\checkmark$ & $\checkmark$ & $\checkmark$ \\
HBV-DNA & $\geq 2000 \mathrm{IU} / \mathrm{mL}$ & $<2000 \mathrm{IU} / \mathrm{mL}$ & Negative \\
ALT & Increased & Normal & Normal \\
Liver damage $^{\text {a }}$ & Yes & No & No \\
\hline
\end{tabular}

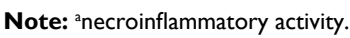

Abbreviations: $H B V$, hepatitis $B$ virus; $H B V-D N A$, hepatitis $B$ genome; $H B s A g$, surface antigen of the hepatitis $B$ virus; $H B$, hepatitis $B ; H B e A G$, envelope antigen of the hepatitis $B$ virus; $H B c$, core antigen of the hepatitis $B$ virus; $A L T$, alanine aminotransferase.

etanercept, golimumab or infliximab. We included in the present review nine out of the ten reports, because one of the identified articles did not specify the anti-TNF- $\alpha$ agent determining HBVr. ${ }^{10}$

\section{$H B V r$ definition}

HBVr can be described as exacerbation of chronic hepatitis B or reactivation of past hepatitis B infection. Several authors, either in patients affected by $\mathrm{CHB}$ or in HBV occult carriers, have used the term reactivation has been used by several authors to refer either to patients affected by $\mathrm{CHB}$, or in $\mathrm{HBV}$ occult carriers. In CHB patients undergoing immunosuppressive agent therapy, HBV replication could noticeably increase because of the impaired cellular and humoral immunity: some authors suggested that this event should not be strictly considered HBVr. ${ }^{11}$ Indeed, once the immunosuppressant treatment is discontinued, the reconstitution of host immunity could flare up the HBV replication as a result of restoration of the cytotoxic activity of the immune cells. ${ }^{12}$ On the other hand, $\mathrm{HBVr}$ can occur in occult carriers, either as a reverse $\mathrm{HBsAg}$ seroconversion (reappearance of $\mathrm{HBsAg}$ ) or as the appearance of HBV-DNA without serum HBsAg positivity.

The clinical course of HBVr could be summarized in three main phases. ${ }^{11,13}$ Initially, it is possible to observe 1) an increment in HBV-DNA levels in the serum of an HBsAg-positive person or 2) the reappearance of HBsAg or HBV-DNA in the serum of a person previously HBsAg negative or with undetectable HBV-DNA. These patients are generally asymptomatic, and serum liver enzymes are within normal ranges. Thereafter, both HBV-DNA and liver enzymes increase in patients' serum, demonstrating liver sufferance, even though patients could still be asymptomatic, especially at the beginning. If $\mathrm{HBVr}$ is diagnosed and properly managed, hepatic injury could resolve either spontaneously or as a result of withholding immunosuppressive therapy or initiation of antiviral therapy. ${ }^{11,13-14}$ 


\section{TNF and HBV}

Experimental studies both in vitro and in vivo have demonstrated that TNF- $\alpha$ is a pivotal cytokine in the biologic process leading to HBV clearance. ${ }^{15,16} \mathrm{TNF}-\alpha$ inhibits HBV replication and stimulates $\mathrm{HBV}$-specific T-cell responses. Consequently, inhibiting TNF- $\alpha$ could enhance HBV replication, allowing the virus to escape the host antiviral defense mechanisms. ${ }^{17}$ Recently, Tzeng et al demonstrated, performing in vitro and in vivo experiments, that HBV viral clearance is not changed in interferon- $\alpha / \beta$ receptor, interleukin-1R knock-out mice, indicating that these innate immunity effectors are not required for $\mathrm{HBV}$ clearance. ${ }^{15} \mathrm{On}$ the contrary, HBV persists in the absence of TNF- $\alpha$ or in mice treated with the soluble TNF- $\alpha$ receptor blocker etanercept. In these mice, there was an increase in PD-1-expressing CD8+ T cells and serum HBV-DNA, HBV core and surface antigen expression, as well as of the viral replication rate within the liver. The induction of TNF- $\alpha$ in clearing HBV has been demonstrated to be dependent on HBV core: TNF- $\alpha$ blockage eliminated HBV core-induced viral clearance effects. Finally, the intrahepatic leukocytes, but not the hepatocytes, are the cell source responsible for TNF- $\alpha$ production induced by HBcAg. These results provide evidence for the TNF- $\alpha-$ mediated innate immune mechanisms in HBV clearance and explain the mechanism of $\mathrm{HBVr}$ in patients undergoing anti-TNF- $\alpha$ agents.

\section{$\mathrm{HBVr}$ risk in psoriatic patients treated with anti-TNFs}

Despite there being a number of reports focusing on $\mathrm{HBVr}$ in gastroenterology and rheumatology patients treated with anti-TNF- $\alpha$, a few authors have reported on HBVr in psoriatic patients. Indeed, most of the studies regard $\mathrm{HBVr}$ in rheumatoid arthritis and anchylosing spondylitis, where PsA is represented with a limited number of patients. In a meta-analysis by Cantini et al, the incidence risk of $\mathrm{HBVr}$ in pooled dermatologic and rheumatic patients was low and ranged from $4.2 \%$ to $6.8 \%$, with higher HBVr recurrences in CHB carriers than in occult carriers. ${ }^{18}$ In clinical practice, antiviral prophylaxis is recommended in CHB (HBsAg+) due to the high risk of $\mathrm{HBVr}$. In contrast, this risk is lower in patients with occult $\mathrm{HBV}$ ( $\mathrm{HBsAg}-$ with anti-HBc+). ${ }^{19}$

Three case series describing a total of 79 psoriatic patients previously exposed to HBV and treated with TNF- $\alpha$ inhibitors did not observe any $\mathrm{HBVr} .^{20-22}$

In 2012, Cho et al conducted a study on seven CHB carrier patients (HBsAg+, $\mathrm{HBeAg}-$ ) affected by Pso or PsA and treated using anti-TNF- $\alpha .{ }^{23}$ The mean duration of anti-TNF- $\alpha$ treatment was 26.6 months. According to the definitions approved by the American Association for the Study of Liver Diseases, five out of seven patients were $\mathrm{HBeAg}-\mathrm{CHB}$ carriers, while the remaining were inactive HBV carriers. Only $1 / 7$ patients that was $\mathrm{HBeAg}+$ for 2 years was treated with a prophylaxis before the anti-TNF- $\alpha$ agent, namely, lamivudine, and thereafter with entecavir. In this cohort, $\mathrm{HBVr}$ was observed in three patients $(42.9 \%$, two CHB and one inactive), even though none had evidence of liver disease. Interestingly, the only patient who received lamivudine prophylaxis was in the HBVr group. ${ }^{23}$

Laurenti et al enrolled eight patients affected by PsA and who were eligible for biologic therapy: one was a CHB $(\mathrm{HBsAg}+)$ and seven were $\mathrm{HBsAg}-/ \mathrm{anti}-\mathrm{HBc}+$. In this study, $\mathrm{HBV}$ screening was performed every 6 months and no $\mathrm{HBVr}$ was observed in a follow-period of up to 3 years. ${ }^{24}$ In 2014, Navarro et al reported of 13 anti-HBc+ patients treated with anti-TNF- $\alpha$ for psoriasis and confirmed the low risk of reactivation, with no HBVr found in the studied population. ${ }^{25}$ Nevertheless, the authors recommended to monitor serum aminotransferase levels, anti-HBs, HBsAg and viral load. In the same year, Morisco et al published a study in which the safety of anti-TNF- $\alpha$ agents was assessed in HBsAg-/anti$\mathrm{HBc}+$ patients ${ }^{26}$ Out of the 220 patients selected for the study, 59 were anti-HBc+, and 40 of them were treated with association of biologics and Disease-modifying antirheumatic drugs (DMARDs). No HBVr was observed and the authors concluded that, in psoriatic patients, immunosuppressive therapy with biologics seems to be safe, regardless of the type of drugs.

In 2015, Sanz-Bueno et al published a retrospective analysis of 20 cases from the Spanish Registry of Adverse Systemic Drug Reactions in Psoriasis (BIOBADADERM) ${ }^{19}$ In the study were also included patients treated with ustekinumab. Out of 1,030 patients treated with biologics, the authors identified 20 anti-HBs+ and anti-HBc+ patients treated with anti-TNF- $\alpha$ agents and none of them had HBVr, therefore assessing the $\mathrm{HBVr}$ risk to be $0 \%$ in their study. All the reported studies are summarized in Table 2.

\section{$\mathrm{HBVr}$ : prevention and management}

Considering the risk of $\mathrm{HBVr}$ in $\mathrm{HBV}$-affected psoriatic patients treated with anti-TNF- $\alpha$ agents and the inefficacy of antiviral treatments in resolving the infection once $\mathrm{HBVr}$ is effective, it is mandatory to consider an $\mathrm{HBVr}$ prophylaxis before therapy.

Moreover, according to the Asian Pacific Association for the Study of the Liver, the American Association for the Study of Liver Diseases and the European Association for the 
Table $2 \mathrm{HBVr}$ in patients with past hepatitis $\mathrm{B}(\mathrm{HBsAg}-)$ and psoriasis in treatment with anti-TNF- $\alpha$

\begin{tabular}{|c|c|c|c|c|c|}
\hline Study & Pts & Prophylaxis & Median follow-up & Anti-TNF & HBV reactivation \\
\hline Nosotti et $\mathrm{al}^{20}$ & 6 & No & 32 months & Eta, Ada & No \\
\hline Cassano et $\mathrm{al}^{22}$ & 62 & No & 48 months & Eta, Ada, Ifx & No \\
\hline Prignano et $\mathrm{al}^{21}$ & II & No & 7.8 months & & No \\
\hline Cho et $\mathrm{al}^{23}$ & 7 & I pt Lam/EntV & 26.6 months & Eta, Ada & 3 reactivations \\
\hline Biondo et $\mathrm{a}^{35}$ & 20 & No & 44 months & Eta, Ada, Ifx, Gol & No \\
\hline Laurenti et $\mathrm{al}^{24}$ & 8 & I pt Lam (HBsAg+) & 3 years & Ada & No \\
\hline Navarro et $\mathrm{al}^{25}$ & 13 & No & 28.6 months & Eta, Ada, Ifx & No \\
\hline Morisco et $\mathrm{al}^{26}$ & 40 & No & 24-72 months & Eta, Ada, Ifx, Gol & No \\
\hline Sanz-Bueno et al ${ }^{19}$ & 20 & No & 40 months & Eta, Ada, Ifx & No \\
\hline
\end{tabular}

Abbreviations: EntV, entecavir; HBV, hepatitis B virus; HBVr, reactivation of HBV; Lam, lamivudine; Pts, patients; TNF, tumor necrosis factor.

Study of the Liver recommendations, all patients, candidates to chemotherapy or immunosuppressive therapy, should be screened for HBV; and for all HBV seronegative patients, who have not been in contact with the virus yet, vaccination must be considered prior to initiating the immunosuppressive therapy. ${ }^{7-9}$ The Asian Pacific Association for the Study of the Liver and the American Association for the Study of Liver Diseases guidelines also recommend prophylactic antiviral therapy in $\mathrm{HBsAg}+$ patients receiving immunosuppressive agents, both during therapy (regardless of HBV-DNA levels) and up to 12 months after therapy discontinuation. ${ }^{7,8}$ Otherwise, the European Association for the Study of the Liver recommendations suggest preemptive therapy for all $\mathrm{HBsAg}+$ patients who are candidates to chemotherapy or immunosuppression (regardless of HBV-DNA levels) during and for 12 months after treatment cessation. ${ }^{9}$ The majority of the published experience with preemptive treatment reports on lamivudine, which may suffice for patients with low $(<2000$ $\mathrm{IU} / \mathrm{mL}$ ) HBV-DNA levels when a finite and short duration of immunosuppression is scheduled (recommendations are shown in Figure 1).

In $\mathrm{HBs} \mathrm{Ag}-/$ anti-HBc+ patients, all authors agree that patients should be treated as HBsAg+ if HBV-DNA is detectable; in case of undetectable HBV-DNA serum, patients should be followed carefully by means of ALT and HBV-DNA testing, regardless of anti-HBs status, and be treated with nucleos(t)ide antagonist therapy upon confirmation of HBVr before ALT elevation. The frequency of monitoring can range from 1 to 3 months, depending on the type of immunosuppressive therapy and comorbidities..$^{7-9}$

The efficacy of antiviral prophylaxis in $\mathrm{HBsAg}+$ patients with hematologic/neoplastic diseases has been established in the literature, since a number of prospective and controlled clinical trials as well as different meta-analyses have clearly shown that preemptive therapy with oral antiviral agents (lamivudine) reduces the risk for $\mathrm{HBVr}$, clinical hepatitis and death. ${ }^{27}$ The nucleos(t)ide analog antiviral drugs lamivudine, adefovir, telbivudine, entecavir and tenofovir may all be of potential use in the prevention of $\mathrm{HBVr}$ in patients undergoing immunosuppression. The majority of reports concern the use of lamivudine or entecavir for the $\mathrm{HBVr}$ prophylaxis. Both drugs appear to reduce the incidence of HBVr in immunosuppressed individuals. ${ }^{28}$

All anti-HBc+/anti-HBs + patients have to be considered as inactive carriers, even without serologic or hepatic evidence of HBV-DNA and, in order to detect possible HBVr,

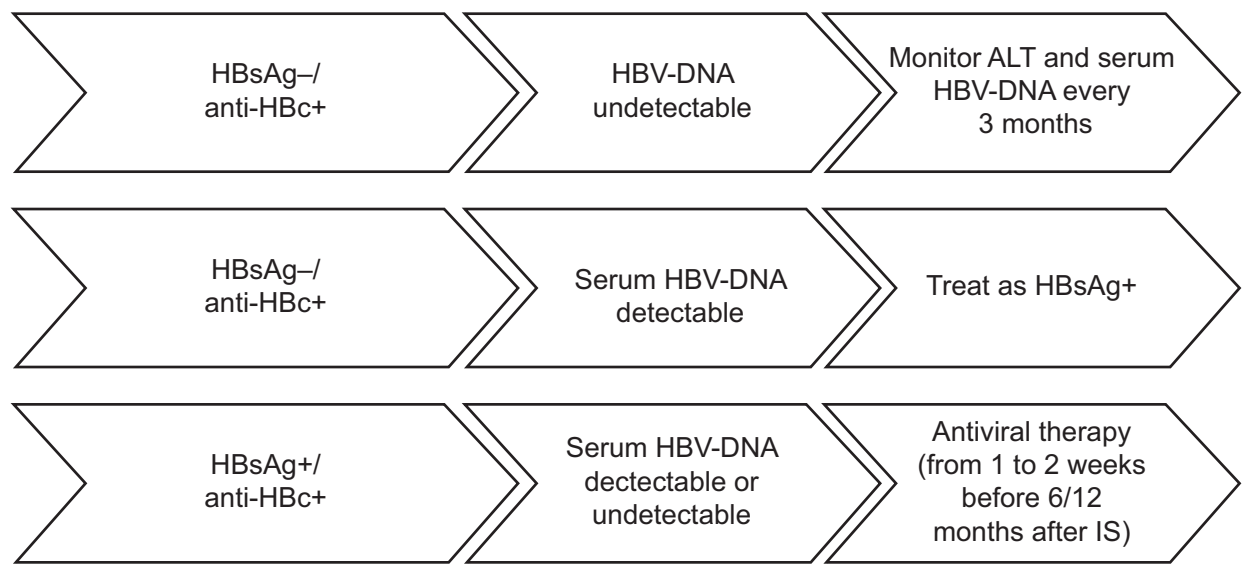

Figure I HBVr prophylaxis: summary of guidelines recommendations.

Abbreviations: ALT, alanine aminotransferase; HBV, hepatitis B virus; HBVr, reactivation of HBV; HBV-DNA, hepatitis B genome; HBsAg, surface antigen of the hepatitis $B$ virus; $H B e A G$, envelope antigen of the hepatitis $B$ virus; $H B c$, core antigen of the hepatitis $B$ virus; IS, immunosuppression. 
liver enzymes and HBV-DNA should be monitored every 3 months and every 6 months, respectively. ${ }^{11,29}$

Hwang and Lok recommend 1) prophylactic antiviral therapy in patients who are deemed to be at high risk of HBVr, 2) prophylactic antiviral therapy or close monitoring and preemptive antiviral therapy in moderate-risk patients if there is evidence of $\mathrm{HBVr}$ and 3) usual medical care in low-risk patients in whom preemptive antiviral therapy is indicated if HBVr occurs. ${ }^{11}$

Concerning the HBVr prophylactic agents, patients given lamivudine during chemotherapy have an $87 \%$ decrease in $\mathrm{HBVr}$ as compared to patients with no prophylaxis. In particular, lamivudine is associated with a $92 \%$ reduction in treatment delays and premature terminations of chemotherapy due to HBV reactivation. ${ }^{30}$ For the treatment of patients with serum HBV-DNA > $2000 \mathrm{IU} / \mathrm{mL}$, either tenofovir or entecavir should be chosen and the therapy should be maintained until they reach the therapeutic end point for CHB. Moreover, entecavir or tenofovir should also be preferred for patients undergoing long-term immunosuppressive therapy. ${ }^{31}$ Notably, entecavir could be a better option for patients with significant renal impairment, as tenofovir has a small risk of inducing proximal tubular dysfunction and renal insufficiency. ${ }^{32-34}$

\section{Conclusion}

As compared to DMARDs (low risk of HBVr) and bone marrow transplantation (high risk of HBVr), anti-TNF- $\alpha$ agents are considered immunosuppressive agents with a moderate risk of $\mathrm{HBVr}^{31}{ }^{31}$ In the last decade, the increasing use of anti-TNF- $\alpha$ therapy for chronic inflammatory diseases has questioned the safety of these agents in patients with serologic signs of HBV infection. More recently, attention has been focused on $\mathrm{HBsAg}-/$ anti-HBc+ subjects. ${ }^{35}$

Although some authors recommend HBV prophylaxis in all anti-HBs+/anti-HBc+ patients undergoing immunosuppressive therapies, HBV prophylaxis should be considered on the basis of type of immunosuppressive agent. Actually, among patients undergoing anti-TNF- $\alpha$ therapy for rheumatoid arthritis, inflammatory bowel diseases or spondyloarthritis, HBVr has been observed in a small proportion of subjects $(6.8 \%)$, while in psoriasis, it has been suggested that the overall assessed risk is lower; hence, HBV prophylaxis should be probably reserved to anti-HBs+/anti-HBc+ patients with a viral load $<2000 \mathrm{IU} / \mathrm{mL}$ and with alterations in serum liver enzymes.

\section{Disclosure}

The authors report no conflicts of interest in this work.

\section{References}

1. Song JE, Kim DY Diagnosis of hepatitis B. Ann Transl Med. 2016; 4(18):338.

2. GBD 2013 Mortality and Causes of Death Collaborators. Global, regional, and national age-sex specific all-cause and cause-specific mortality for 240 causes of death, 1990-2013: a systematic analysis for the Global Burden of Disease Study 2013. Lancet. 2015;10;385(9963):117-171.

3. Ott JJ, Stevens GA, Groeger J, Wiersma ST. Global epidemiology of hepatitis B virus infection: new estimates of age-specific HBsAg seroprevalence and endemicity. Vaccine. 2012;30(12):2212-2219.

4. Domm S, Cinatl J, Mrowietz U. The impact of treatment with tumour necrosis factor-alpha antagonists on the course of chronic viral infections: a review of the literature. Br J Dermatol. 2008;159(6):1217-1228.

5. Wajant H, Pfizenmaier K, Scheurich P. Tumor necrosis factor signaling Cell Death Differ. 2003;10(1):45-65.

6. Marzano A, Angelucci E, Andreone P, et al. Prophylaxis and treatment of hepatitis B in immunocompromised patients. Dig Liver Dis. 2007; 39(5):397-408.

7. Terrault NA, Bzowej NH, Chang KM, Hwang JP, Jonas MM, Murad MH. American Association for the Study of Liver Diseases. AASLD guidelines for treatment of chronic hepatitis B. Hepatology. 2016;63(1):261-283.

8. Sarin SK, Kumar M, Lau GK, et al. Asian-Pacific clinical practice guidelines on the management of hepatitis B: a 2015 update. Hepatol Int. 2016; 10(1):1-98.

9. European Association For The Study Of The Liver. EASL clinical practice guidelines: management of chronic hepatitis B virus infection. J Hepatol. 2012;57(1):167-185.

10. Pérez-Alvarez R1, Díaz-Lagares C, García-Hernández F, et al. Hepatitis $B$ virus (HBV) reactivation in patients receiving tumor necrosis factor (TNF)-targeted therapy: analysis of 257 cases. Medicine (Baltimore). 2011;90(6):359-371.

11. Hwang JP, Lok AS. Management of patients with hepatitis B who require immunosuppressive therapy. Nat Rev Gastroenterol Hepatol. 2014;11(4):209-219.

12. Law MF, Ho R, Cheung CK, et al. Prevention and management of hepatitis $B$ virus reactivation in patients with hematological malignancies treated with anticancer therapy. World J Gastroenterol. 2016;22(28):6484-6500

13. Lok AS, McMahon BJ. Chronic hepatitis B: update 2009. Hepatology. 2009;50:661-662.

14. Hoofnagle JH. Reactivation of hepatitis B. Hepatology. 2009;49: S156-S165.

15. Tzeng HT, Tsai HF, Chyuan IT, et al. Tumor necrosis factor-alpha induced by hepatitis $\mathrm{B}$ virus core mediating the immune response for hepatitis B viral clearance in mice model. PLoS One. 2014;9(7):e103008.

16. Chyuan IT, Tsai HF, Tzeng HT, et al. Tumor necrosis factor-alpha blockage therapy impairs hepatitis B viral clearance and enhances T-cell exhaustion in a mouse model. Cell Mol Immunol. 2015;12(3):317-325.

17. Nathan DM, Angus PW, Gibson PR. Hepatitis B and C virus infections and anti-tumor necrosis factor-alpha therapy: guidelines for clinical approach. J Gastroenterol Hepatol. 2006;21(9):1366-1371.

18. Cantini F, Boccia S, Goletti D, et al. HBV reactivation in patients treated with antitumor necrosis factor-alpha (TNF- $\alpha$ ) agents for rheumatic and dermatologic conditions: a systematic review and meta-analysis. Int $J$ Rheumatol. 2014;2014:926836.

19. Sanz-Bueno J, Vanaclocha F, García-Doval I, et al; And members of the BIOBADADERM group. Risk of reactivation of hepatitis B virus infection in psoriasis patients treated with biologics: a retrospective analysis of 20 cases from the BIOBADADERM database. Actas Dermosifiliogr. 2015;106(6):477-482.

20. Nosotti L, Francesconi F, Izzi S, Berardesca E, Morrone A, Bonifati C. Safety of antitumour necrosis factor- $\alpha$ therapy in psoriatic patients with hepatitis B virus infection. Br J Dermatol. 2010;162(6):1408-1410.

21. Prignano F, Ricceri F, Pescitelli L, Zanieri F, Lotti T. Tumour necrosis factor- $\alpha$ antagonists in patients with concurrent psoriasis and hepatitis B or hepatitis C: a retrospective analysis of 17 patients. Br J Dermatol. 2011;164(3):645-647. 
22. Cassano N, Mastrandrea V, Principi M, et al. Anti-tumor necrosis factor treatment in occult hepatitis $\mathrm{B}$ virus infection: a retrospective analysis of 62 patients with psoriatic disease. J Biol Regul Homeost Agents. 2011;25(2):285-289.

23. Cho YT, Chen CH, Chiu HY, Tsai TF. Use of anti-tumor necrosis factortherapy in hepatitis B virus carriers with psoriasis or psoriatic arthritis: a case series in Taiwan. J Dermatol. 2012;39(3):269-273.

24. Laurenti R, Giovannangeli F, Gubinelli E, et al. Long-term safety of antiTNF adalimumab in $\mathrm{HBc}$ antibody-positive psoriatic arthritis patients: a retrospective case series of 8 patients. Clin Dev Immunol. 2013; 2013:410521.

25. Navarro R, Vilarrasa E, Herranz P, et al. Safety and effectiveness of ustekinumab and antitumour necrosis factor therapy in patients with psoriasis and chronic viral hepatitis $\mathrm{B}$ or $\mathrm{C}$ : a retrospective, multicentre study in a clinical setting. Br J Dermatol. 2013;168(3):609-616.

26. Morisco F, Guarino M, La Bella S, et al. Lack of evidence of viral reac-

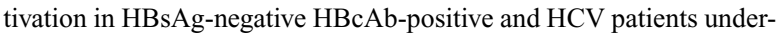
going immunosuppressive therapy for psoriasis. BMC Gastroenterol. 2014;19;14:214.

27. Vassilopoulos D. Should we routinely treat patients with autoimmune/ rheumatic diseases and chronic hepatitis B virus infection starting biologic therapies with antiviral agents? Yes. Eur J Intern Med. 2011; 22(6):572-575.
28. Pattullo V. Prevention of Hepatitis B reactivation in the setting of immunosuppression. Clin Mol Hepatol. 2016;22(2):219-237.

29. Bojito-Marrero L, Pyrsopoulos N. Hepatitis B and Hepatitis C Reactivation in the Biologic Era. J Clin Transl Hepatol. 2014;2(4):240-246.

30. Martyak LA, Taqavi E, Saab S. Lamivudine prophylaxis is effective in reducing hepatitis $\mathrm{B}$ reactivation and reactivation-related mortality in chemotherapy patients: a meta-analysis. Liver Int. 2008;28:28-38.

31. Bessone F, Dirchwolf M. Management of hepatitis B reactivation in immunosuppressed patients: an update on current recommendations. World J Hepatol. 2016;8(8):385-394.

32. Gara N, Zhao X, Collins MT, et al. Renal tubular dysfunction duringlong-term adefovir or tenofovir therapy in chronic hepatitis B. Aliment Pharmacol Ther. 2012; 35:1317-1325.

33. Pipili C, Cholongitas E, Papatheodoridis G. Review article: nucleos(t) ide analogues in patients with chronic hepatitis B virus infection and chronic kidney disease. Aliment Pharmacol Ther. 2014;39:35-46.

34. Marcellin P, Gane E, Buti M, et al. Regression of cirrhosis during treatment with tenofovir disoproxil fumarate for chronic hepatitis B: a 5-year open-label follow-up study. Lancet. 2013;381(9865):468-475.

35. Biondo MI, Germano V, Pietrosanti M, et al. Lack of hepatitis B virus reactivation after anti-tumour necrosis factor treatment in potential occult carriers with chronic inflammatory arthropathies. Eur J Intern Med. 2014;25(5):482-484.
Psoriasis: Targets and Therapy

\section{Publish your work in this journal}

Psoriasis: Targets and Therapy is international, peer-reviewed, open access journal focusing on psoriasis, nail psoriasis, psoriatic arthritis and related conditions, identification of therapeutic targets and the optimal use of integrated treatment interventions to achieve improved outcomes

\section{Dovepress}

and quality of life. Visit http://www.dovepress.com/testimonials.php to read real quotes from published authors. 